\title{
Some Theorems on Integral and Rational Type Contractive Conditions in Dislocated Metric Space
}

\author{
Dinesh Panthi \\ Department of Mathematics, Valmeeki Campus \\ Nepal Sanskrit University, Kathmandu, Nepal. \\ E-mail: panthid06@gmail.com
}

\begin{abstract}
In this article, we establish some coincidence points and common fixed point results on integral and rational type contractive conditions using E.A. and common limit range (CLR) properties in dislocated metric space.
\end{abstract}

Keywords: Dislocated metric, Common fixed point, Weakly compatible maps.

Mathematics Subject Classification: 47H10, 54H25.

\section{Introduction}

In 1922, S. Banach [2] established a fixed point theorem in complete metric space, which is famous now as Banach contraction principle. This principle has been generalized and extended by several authors and has wide applications in the field of pure and applied mathematics. In 2000, P. Hitzler and A.K. Seda [4] obtained a generalization of topology which they named as dislocated topology. The corresponding generalized notion of metric was the dislocated metric. The concept of dislocated metric space was appeared in [8] by S. G. Matthews in 1986 under the name of metric domains. In 2002, A. Branciari [3] obtained a fixed point theorem for a map satisfying contractive condition of integral type with a summable Lebesgue integrable mapping in a complete metric space has been an interesting area of research. B.E Rhoades [9] extended the theorem of Banciari [3] with a most general contractive condition.

The purpose of this paper is to establish some results for integral and rational type contractive conditions for two pairs of maps with E.A. property for coincidence point results and with CLR property for weakly compatible maps for common fixed point results. Our results extend some fixed point theorems in the literature in the setting of dislocated metric space.

\section{Preliminaries}

We start with the following definitions, lemmas and theorems.

Definition 1 [4] Let $X$ be a non empty set and let $d: X \times X \rightarrow[0, \infty)$ be a function satisfying the following conditions:

$$
\begin{aligned}
& \text { 1. } d(x, y)=d(y, x) \\
& \text { 2. } d(x, y)=d(y, x)=0 \text { implies } x=y . \\
& \text { 3. } d(x, y) \leq d(x, z)+d(z, y) \text { for all } x, y, z \in X .
\end{aligned}
$$

Then $d$ is called dislocated metric (or d-metric) on $X$ and the pair $(X, d)$ is called the dislocated metric space (or $d$-metric space). 
Definition 2 [10] Let $A$ and $S$ be two self mappings defined on a metric space $(X, d)$. We say that the mappings $A$ and $S$ satisfy Common Limit Range Property $\left(C L R_{A}\right)$ property if there exists a sequence $\left\{x_{n}\right\} \in X$ such that

$$
\lim _{n \rightarrow \infty} A x_{n}=\lim _{n \rightarrow \infty} S x_{n}=A x
$$

Definition 3 [1] Let $A$ and $S$ be two self mappings defined on a metric space $(X, d)$. We say that the mappings $A$ and $S$ satisfy (E. A.) property if there exists a sequence $\left\{x_{n}\right\} \in X$ such that

$$
\lim _{n \rightarrow \infty} A x_{n}=\lim _{n \rightarrow \infty} S x_{n}=u \text { for some } u \in X .
$$

Definition 4 [6] Let $A$ and $S$ be mappings from a metric space $(X, d)$ into itself. Then, $A$ and $S$ are said to be weakly compatible if they commute at their coincident point; that is, $A x=S x$ for some $x \in X$ implies $A S x=S A x$.

\section{Main Results:}

Now we establish the following result to obtain coincidence point for the given two pairs of mappings using E. A. property.

Theorem 1 Let $(X, d)$ be a dislocated metric space. Let $A, B, S, T: X \rightarrow X$ satisfying the following conditions

$$
\begin{aligned}
& A(X) \subseteq S(X) \text { and } B(X) \subseteq T(X) \\
& \int_{0}^{d(A x, B y)} \phi(t) d t \leq k \int_{0}^{M(x, y)} \phi(t) d t, \quad k \in\left[0, \frac{1}{2}\right)
\end{aligned}
$$

where $\phi: \mathbb{R}^{+} \rightarrow \mathbb{R}^{+}$is a Lebesgue integrable mapping which is summable, non-negative and such that

$$
\begin{aligned}
& \int_{0}^{\epsilon} \phi(t) d t>0 \text { foreach } \epsilon>0 \\
& M(x, y)=\{d(T x, S y) d(T x, A x)+d(S y, A x)+d(T x, S y)+d(T x, A x) \\
& +d(B y, S y)+d(T x, B y)+d(B y, S y) d(A x, S y)\}
\end{aligned}
$$

If the pairs $(A, T)$ or $(B, S)$ satisfy $\mathrm{E}$. A. property and $\mathrm{T}(\mathrm{X})$ is closed then

i) the maps $\mathrm{A}$ and $\mathrm{T}$ have a coincidence point

ii) the maps $\mathrm{B}$ and $\mathrm{S}$ have a coincidence point

Proof: Assume that the pair $(A, T)$ satisfy E.A. property, so there exists a sequence $\left\{x_{n}\right\} \in X$ such that

$$
\lim _{n \rightarrow \infty} A x_{n}=\lim _{n \rightarrow \infty} T x_{n}=u
$$

for some $u \in X$. Since $A(X) \subseteq S(X)$, so there exists a sequence $\left\{y_{n}\right\} \in X$ such that $A x_{n}=S y_{n}$. Hence,

$$
\lim _{n \rightarrow \infty} A x_{n}=\lim _{n \rightarrow \infty} S y_{n}=u
$$

From condition (2) we have

where

$$
\int_{0}^{d\left(A x_{n}, B y_{n}\right)} \phi(t) d t \leq k \int_{0}^{M\left(x_{n}, y_{n}\right)} \phi(t) d t
$$

$$
M\left(x_{n}, y_{n}\right)=\left\{d\left(T x_{n}, S y_{n}\right) d\left(T x_{n}, A x_{n}\right)+d\left(S y_{n}, A x_{n}\right)+d\left(T x_{n}, S y_{n}\right)+d\left(T x_{n}, A x_{n}\right)\right.
$$




$$
\left.+d\left(B y_{n}, S y_{n}\right)+d\left(T x_{n}, B y_{n}\right)+d\left(B y_{n}, S y_{n}\right) d\left(A x_{n}, S y_{n}\right)\right\}
$$

Since,

$$
\lim _{n \rightarrow \infty} d\left(T x_{n}, S y_{n}\right)=\lim _{n \rightarrow \infty} d\left(T x_{n}, A x_{n}\right)=\lim _{n \rightarrow \infty} d\left(S y_{n}, A x_{n}\right)=0
$$

and

$$
\lim _{n \rightarrow \infty} d\left(B y_{n}, S y_{n}\right)=\lim _{n \rightarrow \infty} d\left(T x_{n}, B y_{n}\right)=d\left(B y_{n}, u\right)
$$

Therefore taking limit as $n \rightarrow \infty$ in (7) we get

$$
\begin{aligned}
\operatorname{Lim}_{n \rightarrow \infty} \int_{0}^{d\left(A x_{n}, B y_{n}\right)} \phi(t) d t & \leq \lim _{n \rightarrow \infty} k \int_{0}^{M\left(x_{n}, y_{n}\right)} \phi(t) d t \\
& \leq 2 k \lim _{n \rightarrow \infty} \int_{0}^{d\left(B y_{n}, u\right)} \phi(t) d t
\end{aligned}
$$

that is

$$
\lim _{n \rightarrow \infty} \int_{0}^{d\left(u, B y_{n}\right)} \phi(t) d t \leq 2 k \lim _{n \rightarrow \infty} \int_{0}^{d\left(B y_{n}, u\right)} \phi(t) d t
$$

which is a contradiction, since $k \in\left[0, \frac{1}{2}\right)$.

Hence, $\lim _{n \rightarrow \infty} B y_{n}=u$. Now we have

$$
\lim _{n \rightarrow \infty} A x_{n}=\lim _{n \rightarrow \infty} T x_{n}=\lim _{n \rightarrow \infty} B y_{n}=\lim _{n \rightarrow \infty} S y_{n}=u
$$

Assume $T(X)$ is closed, then there exits $v \in X$ such that $T v=u$. We claim that $A v=u$.

Now from condition (2) we have,

where

$$
\int_{0}^{d\left(A v, B y_{n}\right)} \phi(t) d t \leq k \int_{0}^{M\left(v, y_{n}\right)} \phi(t) d t
$$

$$
\begin{aligned}
M\left(v, y_{n}\right)= & \left\{d\left(T v, S y_{n}\right) d(T v, A v)+d\left(S y_{n}, A v\right)+d\left(T v, S y_{n}\right)+d(T v, A v)\right. \\
& \left.+d\left(B y_{n}, S y_{n}\right)+d\left(T v, B y_{n}\right)+d\left(B y_{n}, S y_{n}\right) d\left(A v, S y_{n}\right)\right\}
\end{aligned}
$$

Since,

$$
\begin{aligned}
& \lim _{n \rightarrow \infty} d\left(S y_{n}, A v\right)=d(u, A v) \\
& \lim _{n \rightarrow \infty} d\left(T v, S y_{n}\right)=\lim _{n \rightarrow \infty} d\left(B y_{n}, S y_{n}\right)=\lim _{n \rightarrow \infty} d\left(T v, B y_{n}\right)=0
\end{aligned}
$$

So, taking limit as $n \rightarrow \infty$ in (8), We conclude that

$$
\int_{0}^{d(A v, u)} \phi(t) d t \leq 2 k \int_{0}^{d(u, A v)} \phi(t) d t
$$

which is a contradiction. Therefore $d(A v, u)=0 \Longrightarrow A v=u$.

Hence,

$$
A v=u=T v .
$$

This proves that $v$ is the coincidence point of $(A, T)$.

Similarly we can show that $v$ is the coincidence point of the pair $(B, S)$.

This completes the proof of our theorem.

On the light of the above theorem we can establish the following corollaries. 
Corrollary 1 Let $(X, d)$ be a dislocated metric space. Let $A, B, S: X \rightarrow X$ satisfying the following conditions

$$
\begin{aligned}
& A(X) \subseteq S(X) \text { and } B(X) \subseteq S(X) \\
& \int_{0}^{d(A x, B y)} \phi(t) d t \leq k \int_{0}^{M(x, y)} \phi(t) d t, \quad k \in\left[0, \frac{1}{2}\right)
\end{aligned}
$$

where $\phi: \mathbb{R}^{+} \rightarrow \mathbb{R}^{+}$is a Lebesgue integrable mapping which is summable, non-negative and such that

$$
\begin{aligned}
\int_{0}^{\epsilon} \phi(t) d t & >0 \text { foreach } \epsilon>0 \\
M(x, y)= & \{d(S x, S y) d(S x, A x)+d(S y, A x)+d(S x, S y)+d(S x, A x) \\
& +d(B y, S y)+d(S x, B y)+d(B y, S y) d(A x, S y)\}
\end{aligned}
$$

If the pairs $(A, S)$ or $(B, S)$ satisfy $\mathrm{E}$. A. property and $\mathrm{S}(\mathrm{X})$ is closed then

i) the maps $\mathrm{A}$ and $\mathrm{S}$ have a coincidence point

ii) the maps B and $\mathrm{S}$ have a coincidence point

Corrollary 2 Let $(X, d)$ be a dislocated metric space. Let $A, S, T: X \rightarrow X$ satisfying the following conditions $A(X) \subseteq S(X)$ and $A(X) \subseteq T(X)$

$$
\int_{0}^{d(A x, A y)} \phi(t) d t \leq k \int_{0}^{M(x, y)} \phi(t) d t, \quad k \in\left[0, \frac{1}{2}\right)
$$

where $\phi: \mathbb{R}^{+} \rightarrow \mathbb{R}^{+}$is a Lebesgue integrable mapping which is summable, non-negative and such that

$$
\begin{aligned}
\int_{0}^{\epsilon} \phi(t) d t & >0 \text { foreach } \epsilon>0 \\
M(x, y)= & \{d(T x, S y) d(T x, A x)+d(S y, A x)+d(T x, S y)+d(T x, A x) \\
& +d(A y, S y)+d(T x, A y)+d(A y, S y) d(A x, S y)\}
\end{aligned}
$$

If the pairs $(A, T)$ or $(A, S)$ satisfy E. A. property and $\mathrm{T}(\mathrm{X})$ is closed then

i) the maps $\mathrm{A}$ and $\mathrm{T}$ have a coincidence point

ii) the maps $\mathrm{A}$ and $\mathrm{S}$ have a coincidence point

Corrollary 3 Let $(X, d)$ be a dislocated metric space. Let $A, S: X \rightarrow X$ satisfying the following conditions $A(X) \subseteq S(X)$

$$
\int_{0}^{d(A x, A y)} \phi(t) d t \leq k \int_{0}^{M(x, y)} \phi(t) d t, \quad k \in\left[0, \frac{1}{2}\right)
$$

where $\phi: \mathbb{R}^{+} \rightarrow \mathbb{R}^{+}$is a Lebesgue integrable mapping which is summable, non-negative and such that

$$
\begin{aligned}
\int_{0}^{\epsilon} \phi(t) d t & >0 \text { foreach } \epsilon>0 \\
M(x, y)= & \{d(S x, S y) d(S x, A x)+d(S y, A x)+d(S x, S y)+d(S x, A x) \\
& +d(A y, S y)+d(S x, B y)+d(A y, S y) d(A x, S y)\}
\end{aligned}
$$

If the pair $(A, S)$ satisfy $\mathrm{E}$. A. property and $\mathrm{S}(\mathrm{X})$ is closed thenthe maps $\mathrm{A}$ and $\mathrm{S}$ have a coincidence point.

Corrollary 4 Let $(X, d)$ be a dislocated metric space. Let $A, B, I: X \rightarrow X$ satisfying the following conditions

$$
A(X) \text { and } B(X) \subseteq I(X)
$$




$$
\int_{0}^{d(A x, B y)} \phi(t) d t \leq k \int_{0}^{M(x, y)} \phi(t) d t, \quad k \in\left[0, \frac{1}{2}\right)
$$

where $\phi: \mathbb{R}^{+} \rightarrow \mathbb{R}^{+}$is a Lebesgue integrable mapping which is summable, non-negative and such that

$$
\begin{aligned}
\int_{0}^{\epsilon} \phi(t) d t & >0 \text { foreach } \epsilon>0 \\
M(x, y)= & \{d(x, y) d(x, A x)+d(y, A x)+d(x, y)+d(x, A x) \\
& +d(B y, y)+d(x, B y)+d(B y, y) d(A x, y)\}
\end{aligned}
$$

If the pairs $(A, I)$ or $(B, I)$ satisfy $\mathrm{E}$. A. property and $\mathrm{T}(\mathrm{X})$ is closed then

i) the maps $A$ and $I$ have a coincidence point

ii) the maps B and I have a coincidence point

Now, we establish the following common fixed point theorem satisfying common limit range property and weakly compatible conditions for two pairs of mappings.

Theorem 2 Let $(X, d)$ be a dislocated metric space. Let $A, B, S, T: X \rightarrow X$ satisfying the following conditions

where,

$$
\begin{aligned}
& A(X) \subseteq S(X) \text { and } B(X) \subseteq T(X) \\
& \int_{0}^{d(A x, B y)} \phi(t) d t \leq k \int_{0}^{M(x, y)} \phi(t) d t, \quad k \in\left[0, \frac{1}{11}\right)
\end{aligned}
$$

$$
\begin{aligned}
M(x, y)= & \left\{\frac{d(T x, S y) d(T x, A x)}{d(T x, B y)}+d(T x, S y)+d(T x, A x)+d(B y, S y)\right. \\
& \left.+d(T x, B y)+d(S y, A x)+\frac{d(B y, S y) d(A x, S y)}{d(T x, B y)}\right\}
\end{aligned}
$$

that

and $\phi: \mathbb{R}^{+} \rightarrow \mathbb{R}^{+}$is a Lebesgue integrable mapping which is summable, non-negative and such

$$
\int_{0}^{\epsilon} \phi(t) d t>0 \text { foreach } \epsilon>0
$$

1. The pairs $(A, T)$ or $(B, S)$ satisfy (CLR)-property

2. The pairs $(A, T)$ and $(B, S)$ are weakly compatible then

i) the maps $\mathrm{A}$ and $\mathrm{T}$ have a coincidence point

ii) the maps $\mathrm{B}$ and $\mathrm{S}$ have a coincidence point

iii) the maps $\mathrm{A}, \mathrm{B}, \mathrm{S}$ and $\mathrm{T}$ have an unique common fixed point.

Proof: Assume that the pair $(A, T)$ satisfy $\left(C L R_{A}\right)$ property, so there exists a sequence $\left\{x_{n}\right\} \in X$ such that

$$
\lim _{n \rightarrow \infty} A x_{n}=\lim _{n \rightarrow \infty} T x_{n}=A x
$$

for some $x \in X$. Since $A(X) \subseteq S(X)$, so there exists a sequence $\left\{y_{n}\right\} \in X$ such that $\lim _{n \rightarrow \infty} A x_{n}=$ $\lim _{n \rightarrow \infty} S y_{n}=A x$. We show that

$$
\lim _{n \rightarrow \infty} B x_{n}=A x
$$

From condition (13) we have

where

$$
\int_{0}^{d\left(A x_{n}, B y_{n}\right)} \phi(t) d t \leq k \int_{0}^{M\left(x_{n}, y_{n}\right)} \phi(t) d t
$$




$$
\begin{aligned}
M\left(x_{n}, y_{n}\right)= & \left\{\frac{d\left(T x_{n}, S y_{n}\right) d\left(T x_{n}, A x_{n}\right)}{d\left(T x_{n}, B y_{n}\right)}+d\left(T x_{n}, S y_{n}\right)+d\left(T x_{n}, A x_{n}\right)+d\left(B y_{n}, S y_{n}\right)\right. \\
& \left.+d\left(T x_{n}, B y_{n}\right)+d\left(S y_{n}, A x_{n}\right)+\frac{d\left(B y_{n}, S y_{n}\right) d\left(A x_{n}, S y_{n}\right)}{d\left(T x_{n}, B y_{n}\right)}\right\}
\end{aligned}
$$

Taking limit as $n \rightarrow \infty$ in (18)we get

$$
\lim _{n \rightarrow \infty} \int_{0}^{d\left(A x_{n}, B y_{n}\right)} \phi(t) d t \leq k \lim _{n \rightarrow \infty} \int_{0}^{M\left(x_{n}, y_{n}\right)} \phi(t) d t,
$$

Since

$$
\begin{aligned}
& \lim _{n \rightarrow \infty} d\left(T x_{n}, S y_{n}\right)=\lim _{n \rightarrow \infty} d\left(T x_{n}, A x_{n}\right)=\lim _{n \rightarrow \infty} d\left(S y_{n}, A x_{n}\right)=0 \\
& \lim _{n \rightarrow \infty} d\left(A x_{n}, B y_{n}\right)=\lim _{n \rightarrow \infty} d\left(A x, B y_{n}\right)=\lim _{n \rightarrow \infty} d\left(B y_{n}, S y_{n}\right)
\end{aligned}
$$

Hence we have

$$
\lim _{n \rightarrow \infty} \int_{0}^{d\left(A x, B y_{n}\right)} \phi(t) d t \leq 2 k \lim _{n \rightarrow \infty} \int_{0}^{d\left(A x, B y_{n}\right)} \phi(t) d t
$$

which is a contradiction, since $k \in\left[0, \frac{1}{11}\right)$.

Therefore,

$$
\lim _{n \rightarrow \infty} d\left(A x, B y_{n}\right)=0 \Longrightarrow \lim _{n \rightarrow \infty} B y_{n}=A x .
$$

Now we have

$$
\lim _{n \rightarrow \infty} A x_{n}=\lim _{n \rightarrow \infty} T x_{n}=\lim _{n \rightarrow \infty} B y_{n}=\lim _{n \rightarrow \infty} S y_{n}=A x
$$

Assume $A(X) \subseteq S(X)$, then there exits $v \in X$ such that $A x=S v$.

We claim that $B v=S v$.

Now from condition (13)

$$
\int_{0}^{d\left(A x_{n}, B v\right)} \phi(t) d t \leq k \int_{0}^{M\left(x_{n}, v\right)} \phi(t) d t
$$

where

$$
\begin{aligned}
M\left(x_{n}, v\right)= & \left\{\frac{d\left(T x_{n}, S v\right) d\left(T x_{n}, A x_{n}\right)}{d\left(T x_{n}, B v\right)}+d\left(T x_{n}, S v\right)+d\left(T x_{n}, A x_{n}\right)+d(B v, S v)\right. \\
& \left.+d\left(T x_{n}, B v\right)+d\left(S v, A x_{n}\right)+\frac{d(B v, S v) d\left(A x_{n}, S v\right)}{d\left(T x_{n}, B v\right)}\right\}
\end{aligned}
$$

Since

$$
\begin{aligned}
& \lim _{n \rightarrow \infty} d\left(T x_{n}, B v\right)=d(A x, B v)=d(S v, B v) \\
& \lim _{n \rightarrow \infty} d\left(T x_{n}, S v\right)=\lim _{n \rightarrow \infty} d\left(T x_{n}, A x_{n}\right)=\lim _{n \rightarrow \infty} d\left(S v, A x_{n}\right)=0
\end{aligned}
$$

So, taking limit as $n \rightarrow \infty$ in (20), we conclude that

$$
\int_{0}^{d(S v, B v)} \phi(t) d t \leq 2 k \int_{0}^{d(S v, B v)} \phi(t) d t
$$

which is a contradiction.

Hence $d(S v, B v)=0 \Longrightarrow S v=B v$.

This proves that $v$ is the coincidence point of of the maps B and $\mathrm{S}$.

Therefore, $S v=B v=A x=w($ Say $)$

Since the pair $(B, S)$ is weakly compatible, so

$$
B S v=S B v \Rightarrow B w=S w
$$

Since $B(X) \subseteq T(X)$ there exists a point $u \in X$ such that $B v=T u$. We show that

$$
T u=A u=w
$$

From condition (13),

where,

$$
\int_{0}^{d(A u, B v)} \phi(t) d t \leq k \int_{0}^{M(u, v)} \phi(t) d t,
$$




$$
\begin{aligned}
M(u, v)= & \left\{\frac{d(T u, S v) d(T u, A u)}{d(T u, B v)}+d(T u, S v)+d(T u, A u)+d(B v, S v)\right. \\
& \left.+d(T u, B v)+d(S v, A u)+\frac{d(B v, S v) d(A u, S v)}{d(T u, B v)}\right\} \\
= & \{d(B v, A u)+d(B v, B v)+d(B v, A u)+d(B v, B v) \\
& +\{d(B v, B v)+d(B v, A u)+d(A u, B v)\} \\
= & \{3 d(B v, B v)+4 d(B v, A u)\} \\
& \leq 10 d(B v, A u) \\
\therefore \quad \int_{0}^{d(A u, B v)} & \phi(t) d t \leq k \int_{0}^{M(u, v)} \phi(t) d t \leq 10 k \int_{0}^{d(A u, B v)} \phi(t) d t
\end{aligned}
$$

which is a contradiction. Hence $d(A u, B v)=0 \Rightarrow A u=B v$.

$$
\therefore A u=B v=T u=w
$$

This proves that $u$ is the coincidence point of the maps $A$ and $T$.

Since the pair $(A, T)$ is weakly compatible so,

$$
A T u=T A u \Rightarrow A w=T w
$$

We show that $A w=w$.

From condition (13)

where

$$
\int_{0}^{d(A w, w)} \phi(t) d t=\int_{0}^{d(A w, B v)} \phi(t) d t \leq k \int_{0}^{M(w, v)} \phi(t) d t,
$$

$$
\begin{aligned}
M(w, v) & =\left\{\frac{d(T w, S v) d(T w, A w)}{d(T w, B v)}+d(T w, S v)+d(T w, A w)+d(B v, S v)\right. \\
& \left.+d(T w, B v)+d(S v, A w)+\frac{d(B v, S v) d(A w, S v)}{d(T w, B v)}\right\} \\
& =\left\{\frac{d(A w, w) d(A w, A w)}{d(A w, w)}+d(A w, w)+d(A w, A w)+d(w, w)\right. \\
& \left.+d(A w, w)+d(w, A w)+\frac{d(w, w) d(A w, w)}{d(A w, w)}\right\} \\
& =\{3 d(A w, w)+2 d(A w, A w)+2 d(w, w)\} \\
& \leq 11 d(A w, w) \\
\therefore \int_{0}^{d(A w, w)} \phi(t) d t & =\int_{0}^{d(A w, B v)} \phi(t) d t \leq k \int_{0}^{M(w, v)} \phi(t) d t \leq 11 k \int_{0}^{d(A w, w)} \phi(t) d t
\end{aligned}
$$

which is a contradiction.

Hence $d(A w, w)=0 \Rightarrow A w=w$. Similarly we obtain $B w=w$.

$\therefore A w=B w=S w=T w=w$. This establishes that $w$ is the common fixed point of four mappings $A, B, S$ and $T$.

\section{Uniqueness:}

let $z(\neq w)$ be other common fixed point of the mappings $A, B, S$ and $T$, then by the condition

where

$$
\int_{0}^{d(w, z)} \phi(t) d t=\int_{0}^{d(A w, B z)} \phi(t) d t \leq k \int_{0}^{M(w, z)} \phi(t) d t
$$




$$
\begin{aligned}
& M(w, z)=\left\{\frac{d(T w, S z) d(T w, A w)}{d(T w, B z)}+d(T w, S z)+d(T w, A w)+d(B z, S z)\right. \\
&\left.+d(T w, B z)+d(S z, A w)+\frac{d(B z, S z) d(A w, S z)}{d(T w, B z)}\right\} \\
&=\left\{\frac{d(w, z) d(w, w)}{d(w, z)}+d(w, z)+d(w, w)+d(z, z)\right. \\
&\left.+d(w, z)+d(z, w)+\frac{d(z, z) d(w, z)}{d(w, z)}\right\} \\
&=3 d(w, z)+2 d(w, w)+2 d(z, z) \\
& \leq 11 d(w, z) \\
& \therefore \int_{0}^{d(w, z)} \phi(t) d t=\int_{0}^{d(A w, B z)} \phi(t) d t \leq k \int_{0}^{M(w, z)} \phi(t) d t \leq 11 k \int_{0}^{d(w, z)} \phi(t) d t
\end{aligned}
$$

which is a contradiction.

Hence, $d(w, z)=0 \Longrightarrow w=z$. This establishes the uniqueness of the common fixed point.

Now, on the light of above theorem we can establish the following corollaries.

Corrollary 5 Let $(X, d)$ be a dislocated metric space. Let $A, B, S: X \rightarrow X$ satisfying the following conditions

$$
\begin{aligned}
& A(X) \subseteq S(X) \text { and } B(X) \subseteq T(X) \\
& \int_{0}^{d(A x, B y)} \phi(t) d t \leq k \int_{0}^{M(x, y)} \phi(t) d t, \quad k \in\left[0, \frac{1}{11}\right)
\end{aligned}
$$

where,

$$
\begin{aligned}
M(x, y) & =\left\{\frac{d(S x, S y) d(S x, A x)}{d(S x, B y)}+d(S x, S y)+d(S x, A x)+d(B y, S y)\right. \\
& \left.+d(S x, B y)+d(S y, A x)+\frac{d(B y, S y) d(A x, S y)}{d(S x, B y)}\right\}
\end{aligned}
$$

and $\phi: \mathbb{R}^{+} \rightarrow \mathbb{R}^{+}$is a Lebesgue integrable mapping which is summable, non-negative and such that

$$
\int_{0}^{\epsilon} \phi(t) d t>0 \text { foreach } \epsilon>0
$$

1. The pairs $(A, S)$ or $(B, S)$ satisfy (CLR)-property

2. The pairs $(A, S)$ and $(B, S)$ are weakly compatible then

i) the maps $\mathrm{A}$ and $\mathrm{S}$ have a coincidence point

ii) the maps $B$ and $S$ have a coincidence point

iii) the maps $\mathrm{A}, \mathrm{B}$ and $\mathrm{S}$ have an unique common fixed point.

Corrollary 6 Let $(X, d)$ be a dislocated metric space. Let $A, S, T: X \rightarrow X$ satisfying the following conditions $A(X) \subseteq S(X)$ and $A(X) \subseteq T(X)$

$$
\int_{0}^{d(A x, A y)} \phi(t) d t \leq k \int_{0}^{M(x, y)} \phi(t) d t, \quad k \in\left[0, \frac{1}{11}\right)
$$

where, 


$$
\begin{aligned}
M(x, y)= & \left\{\frac{d(T x, S y) d(T x, A x)}{d(T x, A y)}+d(T x, S y)+d(T x, A x)+d(A y, S y)\right. \\
& \left.+d(T x, A y)+d(S y, A x)+\frac{d(A y, S y) d(A x, S y)}{d(T x, A y)}\right\}
\end{aligned}
$$

and $\phi: \mathbb{R}^{+} \rightarrow \mathbb{R}^{+}$is a Lebesgue integrable mapping which is summable, non-negative and such that

$$
\int_{0}^{\epsilon} \phi(t) d t>0 \text { foreach } \epsilon>0
$$

1. The pairs $(A, T)$ or $(A, S)$ satisfy (CLR)-property

2. The pairs $(A, T)$ and $(A, S)$ are weakly compatible

then

i) the maps $\mathrm{A}$ and $\mathrm{T}$ have a coincidence point

ii) the maps $\mathrm{A}$ and $\mathrm{S}$ have a coincidence point

iii) the maps $\mathrm{A}, \mathrm{S}$ and $\mathrm{T}$ have an unique common fixed point.

Corrollary 7 Let $(X, d)$ be a dislocated metric space. Let $A, S: X \rightarrow X$ satisfying the following conditions

$$
A(X) \subseteq S(X)
$$

$$
\int_{0}^{d(A x, A y)} \phi(t) d t \leq k \int_{0}^{M(x, y)} \phi(t) d t, \quad k \in\left[0, \frac{1}{11}\right)
$$

where,

$$
\begin{aligned}
M(x, y)= & \left\{\frac{d(S x, S y) d(S x, A x)}{d(S x, A y)}+d(S x, S y)+d(S x, A x)+d(A y, S y)\right. \\
& \left.+d(S x, A y)+d(S y, A x)+\frac{d(A y, S y) d(A x, S y)}{d(S x, A y)}\right\}
\end{aligned}
$$

and $\phi: \mathbb{R}^{+} \rightarrow \mathbb{R}^{+}$is a Lebesgue integrable mapping which is summable, non-negative and such that

$$
\int_{0}^{\epsilon} \phi(t) d t>0 \text { foreach } \epsilon>0
$$

1. The pair $(A, S)$ satisfy (CLR)-property

2. The pair $(A, S)$ is weakly compatible

then, the maps $\mathrm{A}$ and $\mathrm{S}$ have a coincidence point and an unique common fixed point.

Corrollary 8 Let $(X, d)$ be a dislocated metric space. Let $A, B, I: X \rightarrow X$ satisfying the following conditions $A(X)$ and $B(X) \subseteq I(X)$

where,

$$
\int_{0}^{d(A x, B y)} \phi(t) d t \leq k \int_{0}^{M(x, y)} \phi(t) d t, \quad k \in\left[0, \frac{1}{11}\right)
$$

$$
\begin{aligned}
M(x, y) & =\left\{\frac{d(x, y) d(x, A x)}{d(x, B y)}+d(x, y)+d(x, A x)+d(B y, y)\right. \\
& \left.+d(x, B y)+d(y, A x)+\frac{d(B y, y) d(A x, y)}{d(x, B y)}\right\}
\end{aligned}
$$

and $\phi: \mathbb{R}^{+} \rightarrow \mathbb{R}^{+}$is a Lebesgue integrable mapping which is summable, non-negative and such that $\int_{0}^{\epsilon} \phi(t) d t>0$ foreach $\epsilon>0$

1. The pairs $(A, I)$ or $(B, I)$ satisfy (CLR)-property

2. The pairs $(A, I)$ and $(B, I)$ are weakly compatible then

i) the maps A and I have a coincidence point 
ii) the maps B and I have a coincidence point

iii) the maps $\mathrm{A}, \mathrm{B}$ and $\mathrm{I}$ have an unique common fixed point.

\section{Conclusion}

In this article, we used the (E.A.) property to establish coincidence point results and CLR property to claim the existence of common fixed point results of some rational and integral type contraction for two pairs of weakly compatible mappings. Our theorems extend and generalize the theorems of A. Branciary [3], B.E. Rhoades [9], P.Vijayaraju et.al [11] and J. Kumar [7] in the setting of dislocated metric spaces.

\section{Acknowledgment}

This work is carried under the research project SRDIG-76/77-S\&T-09 supported by University Grants Commission, Nepal. The author expresses sincere thank to referees for their careful reading and valuable suggestions for the improvement of manuscript.

\section{References}

[1] Amri, M. and Moutawakil, D. EI.(2002). Some new common fixed point theorems under strict contractive conditions. J. Math. Anal. Appl., 270(1): 181-188.

[2] Banach, S. (1922). Sur les operations dans les ensembles abstraits et leur applications aux equations integrales. Fundamental Mathematicae, 3(7): 133-181.

[3] Branciari, A. (2002). A fixed point theorem for mappings satisfying a general contractive condition of integral Type.International Journal of Mathematics and Mathematical Sciences, 29: 531-536. http://dx.doi.org/10.1155/S0161171202007524

[4] Hitzler, P. and Seda, A. K.(2000). Dislocated topologies, J. Electr. Engg., 51 (12/s): 3-7.

[5] Jungck, G. (1976). Commuting mappings and fixed points, Amer. Math. Monthly. 83: 261-263.

[6] Jungck, G. and Rhoades, B. E.. (1998). Fixed points for set valued functions without continuity, Indian J. Pure Appl. Math., 29(3): 227-238.

[7] Kumar, J. (2013). Common fixed point theorems of weakley compatible maps satisfying E.A .and CLR property.International Journal of Pure and Applied Mathematics,88(3) : 363-376.

[8] Matthews,S. G. (1986).Metric domains for completeness. Technical report 76 [Ph.D. Thesis], Department of Computer Science, University of Warwick, Coventry, UK.

[9] Rhoades,B.E. (2003) .Two fixed point theorems for mappings satisfying a general contractive condition of integral type. International Journal of Mathematics and Mathematical Sciences, 63 : 4007-4013. http://dx.doi.org/10.1155/S0161171203208024

[10]Sintunavarat, W. and Kumam P. ( 2011). Common fixed points for a pair of weakly compatible maps in fuzzy metric spaces, J. Appl. Math., 1-14. https://doi.org/10.1155/2011/637958

[11]Vijayaraju, P., Rhoades, B.E and R Mohan, Raj. (2005). A fixed point theorem for a pair of maps satisfying a general contractive condition of integral type. International Journal of Mathematics and Mathematical Sciences, 15 :2359-2364. 